\title{
Dynamic Response of 3D Frame-Plate Subjected to Wind Load using Finite Element Method
}

\author{
Pham Van Vinh \\ Department of Mechanics \\ Le Quy Don Technical University \\ Hanoi, Vietnam
}

\begin{abstract}
This paper studied the dynamic response of a 3D frame-plate system subjected to wind load. The government equations were established using finite element method. The numerical results were carried out using the Newmark direct integration method. The influence of some parameters on the dynamic response of the system was also investigated and discussed. The numerical results of this work can be useful for calculating, design and examine in practical.
\end{abstract}

Keywords-3D frame-plate, dynamic response, wind load, finite element method.

\section{INTRODUCTION}

The tall building is vulnerable to wind loads due to their structural and aerodynamic characteristics, and their designs are frequently governed by wind loads over design loads. Due to the importance of wind load in tall building design, researchers have made attempts to analyze the dynamic response of the tall building as a complex system of frame and plate. $\mathrm{Gu}$ [1] analyzed the effect of a cross-wind on a typical tall building. Holmes [2] studied the dynamic response of a lattice tower subjected to aerodynamic load with aerodynamic damping. Vibration and motion of a rectangular high-rise building under wind load were investigated by Katagiri [3]. Jeong [4] investigated dynamic response of a tall building subjected to strong wind load using finite element method (FEM) with a frame-plate system model. In [5], Park studied the dynamic response of a high-rise building under wind load with the support of GPS. Kijewski [6] used a full-scale to study the behavior of tall building under wind load with a frame-plate model. Aly [7] studied vibration and vibration control a high-rise building under wind load with passive and active tuned mass damper. Kim [8] and Rosa et al. [9] applied the frame-plate model to investigate the wind-induced excitation control of a tall building with tuned mass dampers. Belloli et al. [10] studied the effects of wind load on a high slender tower using numerical and experimental method. Lin et al. [11] using FEM to investigate characteristics of wind forces acting on a tall building. Mendis et al. [12] studied the dynamic behavior of a tall building subjected to wind load. This paper aims to investigate the dynamic response of a 3D frame-plate system subjected to wind load using FEM.

\section{MODEL AND FINITE ELEMENT FORMULATION}

In this paper, an 8-story 3D frame-plate system under wind load is considered as shown in Fig. 1. Hypothesis: the materials are linear elasticity, the deformation of the system is small, the plate satisfies Reissner-Mindlin plate theory.

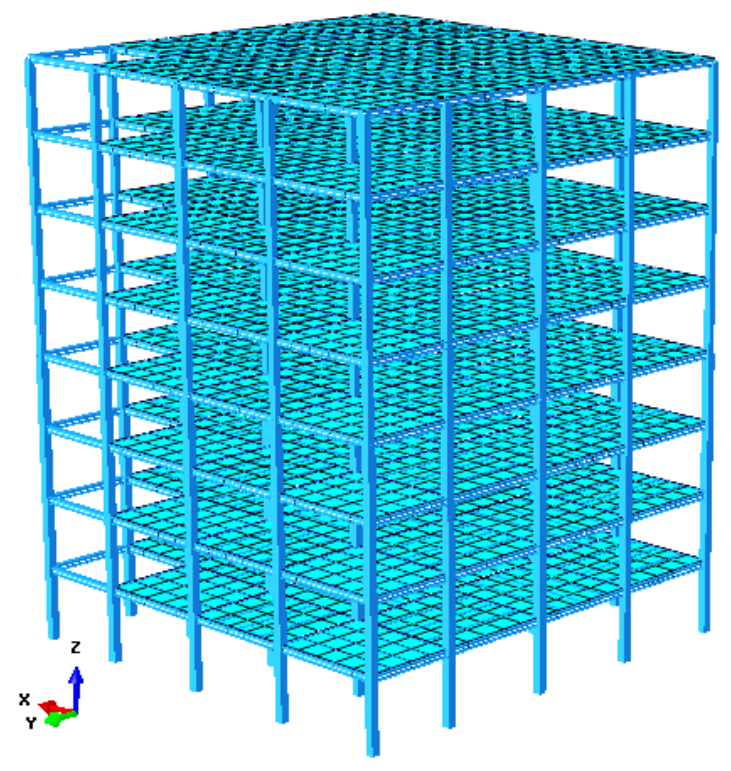

Fig. 1. Model of 3D frame-plate

A. Space beam element

The frame was described by space beam element (3D beam element). The space beam element has 2 nodes and 6 degrees of freedom per node, including $u_{i}, v_{i}, w_{i}, \theta_{x i}, \theta_{y i}, \theta_{z i}$.

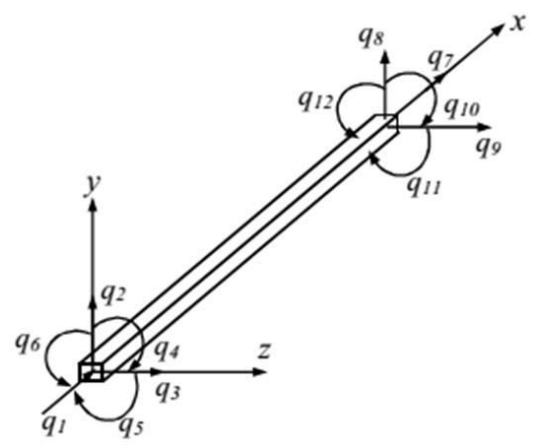

Fig. 2. Space beam element

Displacements in any position point of beam are expressed as follows

$$
\left\{\begin{array}{l}
u(x, y, z)=u_{0}(x)+z \theta_{y}(x)-y \theta_{z}(x) \\
v(x, y, z)=v_{0}(x)-z \theta_{x}(x) \\
w(x, y, z)=w_{0}(x)+y \theta_{x}(x)
\end{array}\right.
$$


where: $u, v$ and $w$ are the displacements along $x, y$ and $z$ directions; $\theta_{x}$ is the rotation of cross-section about the longitudinal axis $x ; \theta_{y}$ and $\theta_{z}$ denote rotations of the cross section about $y$ and $z$ axes.

The strain fields are obtained as following formulas

$$
\begin{aligned}
& \varepsilon_{x}=\frac{\partial u}{\partial x}=\frac{\partial u_{0}}{\partial x}+z \frac{\partial \theta_{y}}{\partial x}-y \frac{\partial \theta_{z}}{\partial x} \\
& \gamma_{x z}=\frac{\partial u}{\partial z}+\frac{\partial w}{\partial x}=\frac{\partial w_{0}}{\partial x}+y \frac{\partial \theta_{x}}{\partial x}+\theta_{y} \\
& \gamma_{x y}=\frac{\partial u}{\partial y}+\frac{\partial v}{\partial x}=\frac{\partial v_{0}}{\partial x}-z \frac{\partial \theta_{x}}{\partial x}-\theta_{z}
\end{aligned}
$$

The nodal displacement vector is

$$
\{q\}_{e}^{b}=\left\{u_{1}, v_{1}, w_{1}, \theta_{x 1}, \theta_{y 1}, \theta_{z 1}, u_{2}, v_{2}, w_{2}, \theta_{x 2}, \theta_{y 2}, \theta_{z 2}\right\}^{T}
$$

where $u_{i}, v_{i}, w_{i}$ are displacement of $i$-th node in $x, y, z$ directions, $\theta_{x i}, \theta_{y i}, \theta_{z i}$ are rotation about $x, y, z$ axes at node $i \quad(i=1,2)$.

The stiffness matrix of $3 \mathrm{D}$ beam element is

$$
[K]_{e}^{b}=\left[K_{x}\right]_{e}^{b}+\left[K_{r}\right]_{e}^{b}+\left[K_{x y}\right]_{e}^{b}+\left[K_{x z}\right]_{e}^{b}
$$

In which $\left[K_{x}\right]_{e}^{b},\left[K_{r}\right]_{e}^{b},\left[K_{x y}\right]_{e}^{b}$ and $\left[K_{x z}\right]_{e}^{b}$ are tension (compression) stiffness matrix, torsion stiffness matrix, bending stiffness matrix in the $x y$ plane, and bending stiffness matrix in the $x z$ plane, respectively.

The mass matrix of the beam element is

$$
[M]_{e}^{b}=\left[M_{x}\right]_{e}^{b}+\left[M_{r}\right]_{e}^{b}+\left[M_{x y}\right]_{e}^{b}+\left[M_{x z}\right]_{e}^{b}
$$

The nodal load vector of the beam element is expressed as

$$
\{f\}_{e}^{b}=\left\{f_{1}, f_{2}, f_{3}, \ldots, f_{10}, f_{11}, f_{12}\right\}^{T}
$$

In the global coordinate

$$
\begin{aligned}
& {\left[K^{\prime}\right]_{e}^{b}=[T]_{e}^{T}[K]_{e}^{b}[T]_{e}} \\
& {\left[M^{\prime}\right]_{e}^{b}=[T]_{e}^{T}[M]_{e}^{b}[T]_{e}} \\
& \left\{f^{\prime}\right\}_{e}^{b}=[T]_{e}^{T}\{f\}_{e}^{b}
\end{aligned}
$$

where $[T]_{e}$ is the coordinate axes transition matrix.

\section{B. Plate element}

According to Reissner-Mindlin plate theory, the displacement fields are written as following formulas

$$
\left\{\begin{array}{l}
u(x, y, z)=u_{0}(x, y)+z \theta_{y}(x, y) \\
v(x, y, z)=v_{0}(x, y)-z \theta_{x}(x, y) \\
w(x, y, z)=w_{0}(x, y)
\end{array}\right.
$$

where $u_{0}, v_{0}, w_{0}$ correspond to displacements of mid-plane, and $\theta_{x}, \theta_{y}$ correspond to rotation angle of the normal section of the plate plane in the survey node.
The bending strains of the plate are obtained as

$$
\left\{\begin{array}{l}
\varepsilon_{x}=\frac{\partial u}{\partial x}=\frac{\partial u_{0}}{\partial x}+z \frac{\partial \theta_{y}}{\partial x} \\
\varepsilon_{y}=\frac{\partial v}{\partial y}=\frac{\partial v_{0}}{\partial y}-z \frac{\partial \theta_{x}}{\partial y} \\
\gamma_{x y}=\frac{\partial u}{\partial y}+\frac{\partial v}{\partial x}=\frac{\partial u_{0}}{\partial y}+\frac{\partial v_{0}}{\partial x}+z\left(\frac{\partial \theta_{y}}{\partial x}-\frac{\partial \theta_{x}}{\partial y}\right)
\end{array}\right.
$$
as

$$
\left\{\begin{array}{l}
\gamma_{x z}=\frac{\partial w}{\partial x}+\frac{\partial u}{\partial z}=\frac{\partial w}{\partial x}+\theta_{y} \\
\gamma_{y z}=\frac{\partial w}{\partial y}+\frac{\partial v}{\partial z}=\frac{\partial w}{\partial y}-\theta_{x}
\end{array}\right.
$$

The linear elastic stress-strain relationship of plate is defined as

$$
\sigma_{b}=[D]_{b} \varepsilon_{b}, \sigma_{s}=[D]_{s} \varepsilon_{s}
$$

where

$$
\begin{gathered}
{[D]_{b}=\frac{E}{1-v^{2}}\left[\begin{array}{ccc}
1 & v & 0 \\
v & 1 & 0 \\
0 & 0 & \frac{1-v}{2}
\end{array}\right]} \\
{[D]_{s}=G\left[\begin{array}{ll}
1 & 0 \\
0 & 1
\end{array}\right]}
\end{gathered}
$$

with $G=\frac{E}{2(1-v)}$ is shear modulus of material.

In this paper, the plate is discretized by quadrilateral 4node plate element, each node contains 6 degrees of freedom as in Fig. 3. The displacement and rotations in the element are interpolated from the nodal values as

$$
\left\{\begin{array}{l}
u=\sum_{i=1}^{4} N_{i}(\xi, \eta) u_{i} \\
v=\sum_{i=1}^{4} N_{i}(\xi, \eta) v_{i} \\
w=\sum_{i=1}^{4} N_{i}(\xi, \eta) w_{i} \\
\theta_{x}=\sum_{i=1}^{4} N_{i}(\xi, \eta) \theta_{x i} \\
\theta_{y}=\sum_{i=1}^{4} N_{i}(\xi, \eta) \theta_{y i} \\
\theta_{z}=\sum_{i=1}^{4} N_{i}(\xi, \eta) \theta_{z i}
\end{array}\right.
$$


where $N_{i}(s, t)$ are the shape functions of the element in the local coordinate system, which is determined by the coordinates of the element node as

$$
N_{i}(s, t)=\frac{1}{4}\left(1+\xi_{i} \xi\right)\left(1+\eta_{i} \eta\right), i=\overline{1,4}
$$

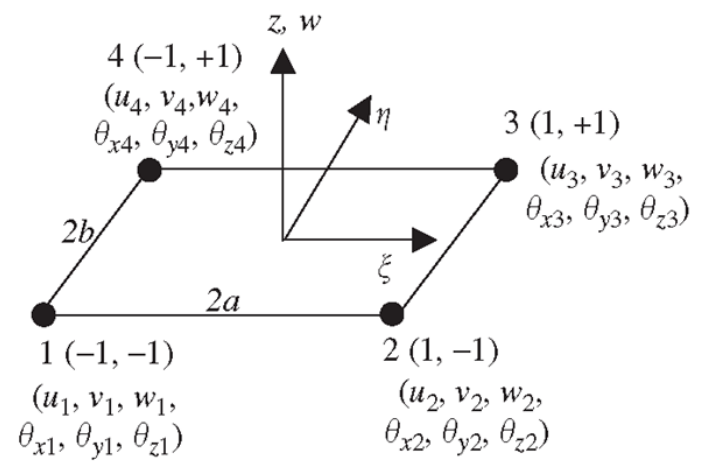

Fig. 3. Four-node plate element

The element node displacement $\{q\}_{e}^{p}$ is written as

$$
\{q\}_{e}^{p}=\left\{u_{1}, v_{1}, w_{1}, \theta_{x 1}, \theta_{y 1}, \theta_{z 1}, \ldots, u_{4}, v_{4}, w_{4}, \theta_{x 4}, \theta_{y 4}, \theta_{z 4}\right\}^{T}
$$

Strains of the element are defined as

$$
\varepsilon_{b}=z[B]_{b}\{q\}_{e}^{p}, \varepsilon_{s}=[B]_{s}\{q\}_{e}^{p}
$$

where $B_{b}, B_{s}$ are strain-displacement matrices for bending and shear contributions which are obtained by derivation of the shape functions as

$$
\begin{gathered}
{[B]_{b}=\left[\begin{array}{ccccccc}
0 & \frac{\partial N_{1}}{\partial x} & 0 & \ldots & 0 & \frac{\partial N_{4}}{\partial x} & 0 \\
0 & 0 & \frac{\partial N_{1}}{\partial y} & \ldots & 0 & 0 & \frac{\partial N_{4}}{\partial y} \\
0 & \frac{\partial N_{1}}{\partial y} & \frac{\partial N_{1}}{\partial x} & \ldots & 0 & \frac{\partial N_{4}}{\partial y} & \frac{\partial N_{4}}{\partial x}
\end{array}\right]} \\
{[B]_{s}=\left[\begin{array}{ccccccc}
\frac{\partial N_{1}}{\partial x} & N_{1} & 0 & \ldots & \frac{\partial N_{1}}{\partial x} & N_{4} & 0 \\
\frac{\partial N_{1}}{\partial y} & 0 & N_{1} & \ldots & \frac{\partial N_{1}}{\partial y} & 0 & N_{4}
\end{array}\right]}
\end{gathered}
$$

The train energy of a plate element is given by

$$
\Pi_{e}=\frac{1}{2} \int_{V_{e}} \varepsilon_{b}^{T} D_{b} \varepsilon_{b} d V+\frac{\mu}{2} \int_{V_{e}} \varepsilon_{s}^{T} D_{s} \varepsilon_{s} d V
$$

where $\mu$ is the shear correction factor, in general $\mu=5 / 6$.

The element stiffness matrix is given as

$$
\begin{aligned}
& {[K]_{e}^{p}=[K]_{e b}^{p}+[K]_{e s}^{p}} \\
& =\frac{1}{2} \int_{V_{e}}[B]_{b}^{T} D_{b}[B]_{b} d V+\frac{\mu}{2} \int_{V_{e}}[B]_{s}^{T} D_{s}[B]_{s} d V
\end{aligned}
$$

The kinetic energy of a plate element is given by

$$
T_{e}=\frac{1}{2}\{\dot{q}\}_{e}^{p T} \int_{V_{e}} \rho[N]^{T}[N] d V\{\dot{q}\}_{e}^{p}
$$

The mass matrix of the plate element is expressed as

$$
[M]_{e}^{p}=\int_{V_{e}} \rho[N]^{T}[N] d V
$$

The nodal load vector of the plate element is expressed as

$$
\{f\}_{e}^{b}=\left\{f_{1}, f_{2}, f_{3}, \ldots, f_{22}, f_{23}, f_{24}\right\}^{T}
$$

In the global coordinate

$$
\begin{aligned}
& {\left[K^{\prime}\right]_{e}^{p}=[T]_{e}^{T}[K]_{e}^{p}[T]_{e}} \\
& {\left[M^{\prime}\right]_{e}^{p}=[T]_{e}^{T}[M]_{e}^{p}[T]_{e}} \\
& \left\{f^{\prime}\right\}_{e}^{p}=[T]_{e}^{T}\{f\}_{e}^{p}
\end{aligned}
$$

where $[T]_{e}$ is the coordinate axes transition matrix.

\section{Wind load}

Under the influence of wind load, each plate element is affected by distributed lift force $L_{w}$ and distributed bending moment $M_{w}$, which are obtained as

$$
\begin{aligned}
& L_{w}=\frac{1}{2} \rho_{a} U^{2} B\left[K H_{1}^{*}(K) \frac{\dot{h}}{U}+K H_{2}^{*}(K) \frac{B \dot{\alpha}}{U}+K^{2} H_{3}^{*}(K) \alpha\right] \\
& M_{w}=\frac{1}{2} \rho_{a} U^{2} B^{2}\left[K A_{1}^{*}(K) \frac{\dot{h}}{U}+K A_{2}^{*} \frac{B \dot{\alpha}}{U}+K^{2} A_{3}^{*}(K) \alpha\right]
\end{aligned}
$$

where $\rho_{a}$ is air density, $U$ is wind velocity, $B$ is the area of cross-section, $K$ is expressed as $K=B \omega / U$.

The functions $A_{i}^{*}(K), H_{i}^{*}(K)$ are calculated as

$$
\begin{aligned}
& H_{1}^{*}(K)=-\frac{\pi}{k} F(k) \\
& H_{2}^{*}(K)=-\frac{\pi}{4 k}\left[1+F(k)+\frac{2 G(k)}{k}\right] \\
& H_{3}^{*}(K)=-\frac{\pi}{2 k^{2}}\left[F(k)-\frac{k G(k)}{2}\right] \\
& A_{1}^{*}(K)=\frac{\pi}{4 k} F(k) \\
& A_{2}^{*}(K)=-\frac{\pi}{16 k}\left[1-F(k)-\frac{2 G(k)}{k}\right] \\
& A_{3}^{*}(K)=\frac{\pi}{8 k^{2}}\left[\frac{k^{2}}{8}+F(k)-\frac{k G(k)}{2}\right]
\end{aligned}
$$

where

$$
\begin{aligned}
& k=K / 2 \\
& F(k)=\frac{0.500502 k^{3}+0.5160 k^{2}+0.2104 k+0.02157}{k^{3}+1.035378 k^{2}+0.251293 k+0.021508} \\
& G(k)=-\frac{0.000146 k^{3}+0.1224 k^{2}+0.3272 k+0.00199}{k^{3}+2.481481 k^{2}+0.93453 k+0.089318}
\end{aligned}
$$




\section{Government equation of motion}

The Hamilton principle is applied to obtain the equation of motion

$$
\delta H_{e}=\delta \int_{t 0}^{t 1}\left(T_{e}-\Pi_{e}+W_{e}\right) d t=0
$$

The differential equation of motion of element can be obtained as

$$
\begin{aligned}
{[M]_{e}\{\ddot{q}\}_{e} } & +\left([C]_{e}+[C]_{e}^{a r}\right)\{\dot{q}\}_{e} \\
& +\left([K]_{e}+[K]_{e}^{a r}\right)\{q\}_{e}=\{F\}_{e}
\end{aligned}
$$

The equation of motion of $3 \mathrm{D}$ frame-plate system is represented as follows

$$
[M]\{\ddot{q}\}+[C]\{\dot{q}\}+[K]\{q\}=\{F\}
$$

where $[M],[C]=[C]^{s y s}+[C]^{a r},[K]=[K]^{s y s}+[K]^{a r}$ are the mass, damping and stiffness matrices of the system, respectively. They are obtained by assembling all its element matrix through the direct stiffness method and imposing the prescribed boundary conditions. The damping matrix is constructed by using the Rayleigh damping theory in following form:

$$
[C]=a[M]+b[K]+[C]^{a r}
$$

with $a$ and $b$ are calculated by using damping ratio $\xi$ and natural frequencies $\omega_{1}, \omega_{2}$ as following formula

$$
\left\{\begin{array}{l}
a \\
b
\end{array}\right\}=\frac{2 \zeta}{\omega_{1}+\omega_{2}}\left\{\begin{array}{c}
\omega_{1} \omega_{2} \\
1
\end{array}\right\}
$$

This is a secondary linear differential equation with timedependent coefficients. This equation is integrated using the Newmark direct integration method. The average constant acceleration method is $\alpha=0.25$ and $\beta=0.5$ which ensures numerically unconditional stability is used.

\section{E. Finite element algorithm}

Step 1: Calculate the mass, damping, stiffness matrices and force vector.

Step 2: Set initial conditions.

Step 3: Calculate initial parameters.

Step 4: Conduct inter cycle in each time step,

- Update the damping, stiffness matrices and force vector,

- Calculate vector of displacement, velocity, acceleration responses using following formulas

$$
\begin{aligned}
& \{q\}_{i+1}=\left[\frac{1}{\alpha(\Delta t)^{2}}[M]+\frac{\beta}{\alpha \Delta t}[C]+[K]\right]^{-1} \\
& \quad \times\left\{\{F\}+[M]\left[\frac{\{q\}_{i}}{\alpha(\Delta t)^{2}}+\frac{\{\dot{q}\}_{i}}{\alpha \Delta t}+\left(\frac{1}{2 \alpha}-1\right)\{\ddot{q}\}_{i}\right]\right. \\
& \left.\quad+[C]\left[\frac{\beta\{q\}_{i}}{\alpha \Delta t}+\left(\frac{\beta}{\alpha}-1\right)\{\dot{q}\}_{i}+\left(\frac{\beta}{\alpha}-2\right) \frac{\Delta t\{\ddot{q}\}_{i}}{2}\right]\right\}
\end{aligned}
$$

$$
\begin{gathered}
\{\ddot{q}\}_{i+1}=\frac{\{q\}_{i+1}-\{q\}_{i}}{\alpha(\Delta t)^{2}}-\frac{\{\dot{q}\}_{i}}{\alpha \Delta t}-\left(\frac{1}{2 \alpha}-1\right)\{\ddot{q}\}_{i} \\
\{\dot{q}\}_{i+1}=\{\dot{q}\}_{i}+(1-\beta) \Delta t\{\ddot{q}\}_{i}+\beta \Delta t\{\ddot{q}\}_{i+1}
\end{gathered}
$$

Step 5: Export and display results.

\section{RESULTS AND DISCUSSION}

The 3D frame-plate system including 25 vertical columns, 80 beams and 8 floors as shown in Fig. 1. For each story has a height of $h=3 \mathrm{~m}$, total height level of the building $H=24 \mathrm{~m}$ width $W=20 m$, depth $B=20 m$, cross-section of column is $0.5 m \times 0.5 m$, beams are $0.2 m \times 0.3 m$, and floors' thickness is $0.15 \mathrm{~m}$. Columns and floors are made of concrete with properties of $E=3.52 \cdot 10^{10} \mathrm{~N} / \mathrm{m}^{2}, \quad \rho=2.80 \cdot 10^{3} \mathrm{~kg} / \mathrm{m}^{3}$, $v=0.3$. The end of 25 vertical columns are clamped. The velocity of wind is $50 \mathrm{~m} / \mathrm{s}$.

Results export: the central point of $8^{\text {th }}$ floor (point A), the central point of $4^{\text {th }}$ floor (point B). The horizontal acceleration, velocity and displacement of point $\mathrm{A}$ and $\mathrm{B}$ are shown in Fig. $4-$ Fig. 6.

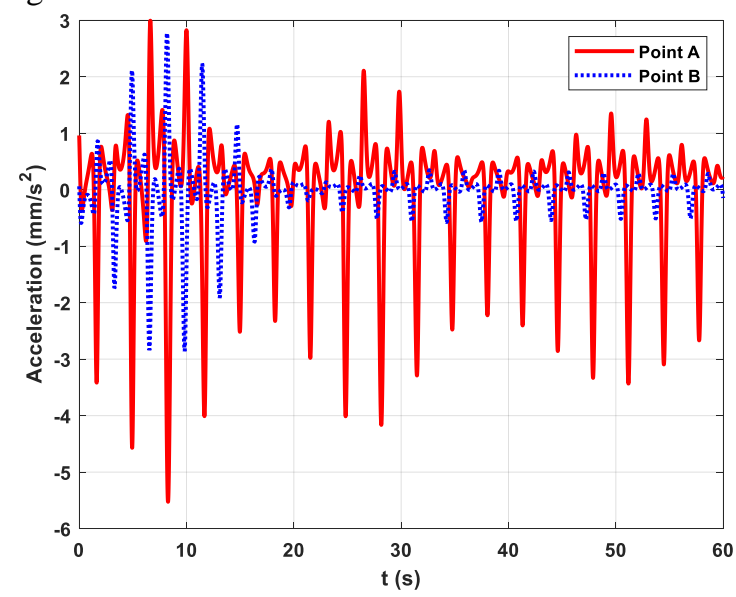

Fig. 4. The acceleration of point A and B

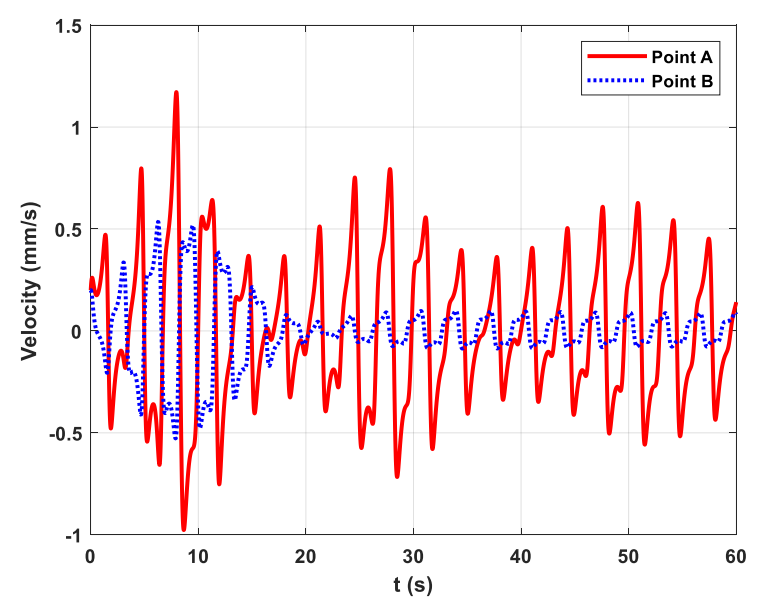

Fig. 5. The velocity of point A and B 


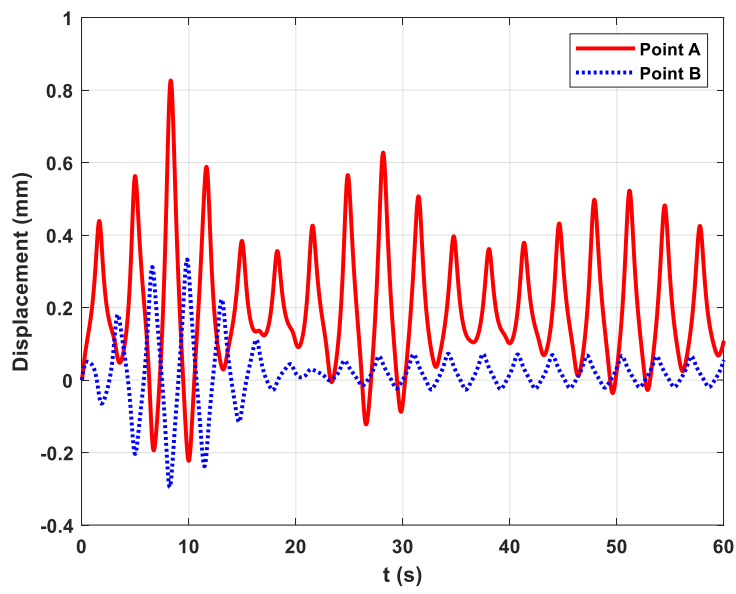

Fig. 6. The displacement of point A and B

According to Fig. 4 - Fig. 6, when the system subjected to wind load, it deforms and vibrates. The horizontal acceleration, velocity and displacement of point $\mathrm{A}$ is larger than point $\mathrm{B}$.

\section{A. Influence of the system height}

The relationship between the system height $H$ and the displacement of point $\mathrm{A}$ and $\mathrm{B}$ are shown in Fig. 7. It shows that the displacement of the system has a nonlinear dependence on the height of the system. The displacement of point $A$ and $B$ increase when increasing the height of the system. The displacement of point $\mathrm{A}$ increases faster than the displacement of point B, so when increasing the system height, the upper points will fluctuate greatly.

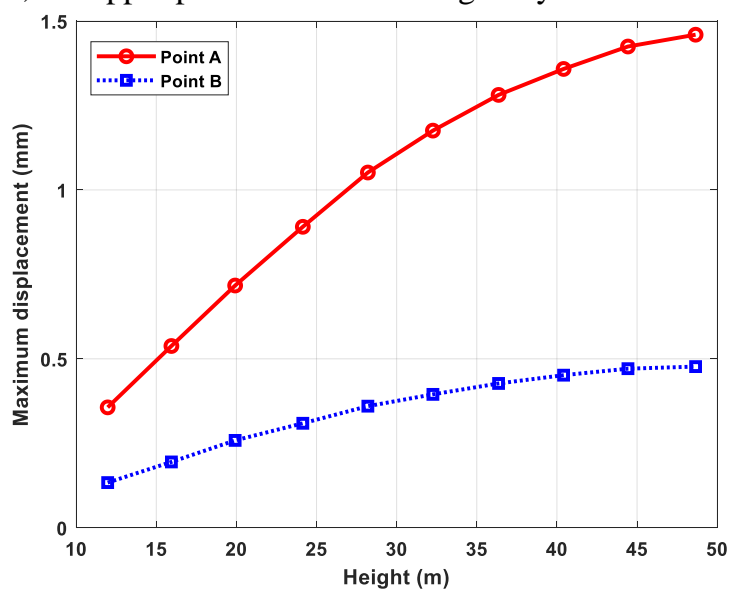

Fig. 7. The maximum displacement of point $\mathrm{A}$ and $\mathrm{B}$ depend on the height

\section{B. Influence of the wind velocity}

Influence of the wind velocity is shown in Figure 6. The velocity of wind has a great effect on the deflection of the system. It shows that the displacement of point A and B increase when increasing the wind velocity. When the wind velocity is slow, the displacement increases slowly then increases quickly when the wind velocity is large. Therefore, it can be seen that the strong wind greatly affects the behavior of the frame-plate system.

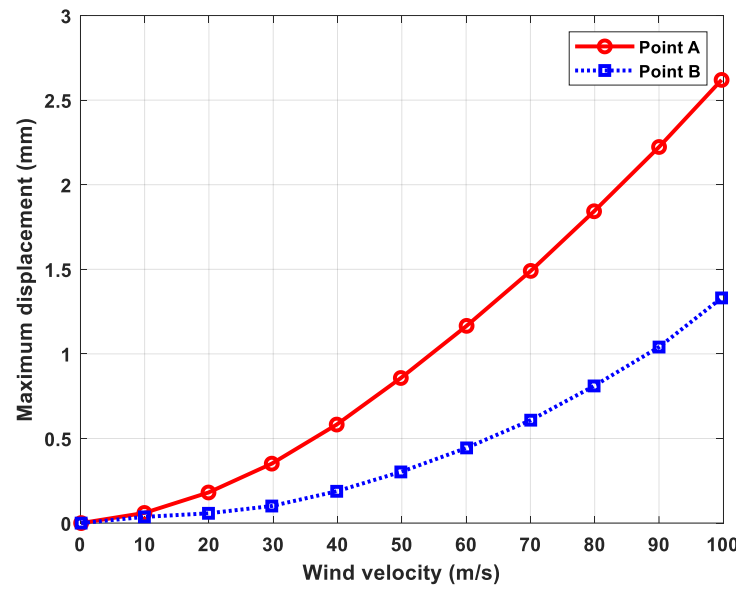

Fig. 8. The maximum displacement of point $\mathrm{A}$ and $\mathrm{B}$ depend on the velocity

\section{CONCLUSION}

In this study, a 3D model of a frame-plate system was developed and investigated. The algorithm and dynamic analysis program were established based on finite element method and Newmark direct integration method. The numerical results show that the wind load has a great effect on the dynamic response of model and can cause the dangerous status of the system. The results of this paper can be useful for calculation, design and selection of reasonable solutions for new design and reinforcement of modern tall buildings.

\section{REFERENCES}

[1] Gu, M., and Y. Quan, "Across-wind loads on typical tall buildings", Journal of Wind Engineering \& Industrial Aerodynamics., vol. 92, pp. $1147-1165,2004$

[2] J. D. Holmes, “Along-wind response of lattice towers. II: Aerodynamic damping and deflection”, Engineering Structures, vol. 18, pp. 483-488, 1996

[3] J. Katagiri, T. Ohkuma, H. Marukawa, and S. Shimomura, "Motioninduced wind forces acting on rectangular high-rise buildings with side ratio of 2." Journal of Structural and Construction Engineering, vol. 65 pp. 25-32, 2000 .

[4] U. Y. Jeong, "Advances in tall building design under strong winds", In Structures Congress 2015. Reston, VA: ASCE/SEI, 2015.

[5] H. S. Park, H. G. Sohn, I. S. Kim, and J. H. Park, "Application of GPS to monitoring of wind-induced responses of high-rise buildings," The Structural Design of Tall and Special Buildings, vol. 17, no. 1, pp. $117-$ 132,2008

[6] T. Kijewski, "Full-scale study of the behavior of tall buildings under winds," Proc. SPIE, vol. 4337, no. 219, pp. 441-450, 2001

[7] A. M. Aly, "Vibration control of high-rise buildings for wind: A robust passive and active tuned mass damper," Smart Structures and Systems, vol. 13, no. 3, pp. 473-500, 2014.

[8] Y.-M. Kim, K.-P. You, and H.-Y. Kim, "Wind-induced excitation control of a tall building with tuned mass dampers," The Structural Design of Tall and Special Buildings, vol. 17, no. 3, pp. 669-682, 2008

[9] L. Rosa, G. Tomasini, A. Zasso, and A. M. Aly, "Wind-induced dynamics and loads in a prismatic slender building: A modal approach based on unsteady pressure measurements," Journal of Wind Engineering \& Industrial Aerodynamics, vol. 107-108, pp. 118-130, 2012.

[10] M. Belloli, L. Rosa, and A. Zasso, "Wind loads on a high slender tower: Numerical and experimental comparison," Engineering Structures, vol. 68, pp. 24-32, 2014

[11] N. Lin, C. Letchford, Y. Tamura, B. Liang, and O. Nakamura, "Characteristics of wind forces acting on tall buildings," Journal of Wind Engineering \& Industrial Aerodynamics, vol. 93, no. 3, pp. 217 $242,2005$. 